\title{
Prehospital rapid sequence induction by emergency physicians: Is it safe?
}

\author{
C A Mackay, J Terris, T J Coats
}

\begin{abstract}
Objectives-To determine if there were differences in practice or intubation mishap rate between anaesthetists and accident and emergency physicians performing rapid sequence induction of anaesthesia (RSI) in the prehospital setting.
\end{abstract}

Methods-All patients who underwent RSI by a Helicopter Emergency Medical Service (HEMS) doctor from 1 May 1997 to 30 April 1999 were studied by retrospective analysis of in-flight run sheets. Intubation mishaps were classified as repeat attempts at intubation, repeat drug administration and failed intubation.

Results-RSI was performed on 359 patients by 10 anaesthetists (202 patients) and nine emergency physicians (157 patients). Emergency physicians recorded a larger number of patients as having Cormack and Lehane grade 3 or 4 laryngoscopy than anaesthetists $(p<0.0001)$ but were less likely to use a gum elastic bougie to assist intubation $(p=0.024)$. Patients treated by emergency physicians did not have a significantly different pulse, blood pressure, oxygen saturation or end tidal $\mathrm{CO}_{2}$ to patients treated by anaesthetists at any time after intubation. Emergency physicians were more likely to anaesthetise patients with a Glasgow Coma Score $>12$ than anaesthetists $(p=0.003)$. There were two failed intubations $(1 \%)$ in the anaesthetist group and four $(2.5 \%)$ in the emergency physician group. Repeat attempts at intubation and repeat drug administration occurred in $<2 \%$ of each group.

Conclusions-RSI performed by emergency physicians was not associated with a significantly higher failure rate or an increased number of intubation mishaps than RSI performed by anaesthetists. Emergency physicians were able to safely administer sedative and neuromuscular blocking drugs in the prehospital situation. It is suggested that emergency physicians can safely perform rapid sequence induction of anaesthesia and intubation. (Emerg Med f 2001;18:20-24)

Keywords: rapid sequence intubation

Airway management is a priority in the resuscitation of the trauma patient. Endotracheal intubation provides optimal airway control but often requires the use of sedative and muscle relaxant drugs in patients with intact upper airway reflexes. ${ }^{12}$ Attempted intubation without these drugs may lead to a rise in intracranial pressure during laryngoscopy, which should be avoided in patients with head injury. ${ }^{34}$

Rapid sequence induction of anaesthesia and intubation (RSI) have been shown to be safe when performed by emergency physicians in hospital ${ }^{5}{ }^{6}$ but are predominantly carried out by anaesthetists in most UK accident and emergency (A\&E) departments. Prehospital RSI is performed by aeromedical teams in the $\mathrm{USA}^{7-10}$ but literature on prehospital anaesthesia in Britain is limited. ${ }^{112}$

The Helicopter Emergency Medical Service (HEMS) attends to trauma patients in the Greater London area and provides a doctor capable of performing rapid sequence induction of anaesthesia and advanced airway skills. Doctors working for HEMS in recent years have a background of either A\&E or anaesthesia and are at the end of specialist registrar training.

Our aim was to study prehospital rapid sequence induction of anaesthesia to determine if there were differences in practice between anaesthetists and $A \& E$ physicians and to document difficulties encountered and intubation mishaps.

\section{Methods}

A retrospective review of case notes was carried out to identify all patients who underwent RSI from 1 May 1997 to 30 April 1999. Patients who did not receive an induction agent or muscle relaxant were excluded from the analysis.

POPULATION AND SETTING

HEMS is based at the Royal London Hospital and is tasked by paramedics on the special incident desk at the London Ambulance Service control room. ${ }^{13}$ The flight crew consist of two pilots, a doctor and a paramedic. The majority of primary missions attended are trauma related and occur within the boundaries of the M25 motorway, although occasional requests to assist outer county ambulance crews occur. On scene, the doctor is responsible for assessing and resuscitating the patient who is then taken to the nearest or quickest, most appropriate hospital that has all the necessary facilities on site.

All doctors undergo a training month where a more experienced HEMS colleague or consultant at each mission attended supervises them. Any specific additional training requirements are tackled during this period. Standard Operating Procedures are issued to all doctors that are guidelines on patient management. These guidelines suggest that rapid sequence induction and intubation should be considered 
Table 1 Rapid sequence induction procedure

Preoxygenate with $100 \%$ oxygen

Monitor pulse, BP, ECG and oxygen saturation

Establish intravenous access

Loosen cervical collar and maintain in-line cervical immobilistaion from below

Give IV bolus etomidate $(0.3 \mathrm{mg} / \mathrm{kg})$

Give IV bolus suxamethonium $(1-1.5 \mathrm{mg} / \mathrm{kg})$

Apply cricoid pressure at the time of injection of induction agent

Once muscle fasciculations have stopped, perform laryngoscopy and intubate using

predetermined sized endotracheal tube cut to appropriate length

Verify tube postion by auscultation over both sides of the chest and over the stomach

and with attached end tidal $\mathrm{CO}_{2}$ monitoring

Remove cricoid pressure and replace cervical collar

Secure endotracheal tube

Administer pancuronium $(0.1 \mathrm{mg} / \mathrm{kg})$ to maintain paralysis for transport

Administer propofol (1-2 mg/kg), midazolam $(0.1-0.4 \mathrm{mg} / \mathrm{kg})$ or morphine $(0.1$ $\mathrm{mg} / \mathrm{kg}$ ) for sedation

Table 2 Clinical indication for rapid sequence induction

\begin{tabular}{lll}
\hline & Anaesthetists & $\begin{array}{c}\text { Emergency } \\
\text { physicians }\end{array}$ \\
\hline Isolated head injury & 89 & 71 \\
Isolated chest injury & 4 & 3 \\
Multisystem trauma with head injury & 85 & 64 \\
Multisystem trauma without head injury & 7 & 12 \\
Burn & 8 & 6 \\
Other & 9 & 1 \\
\hline
\end{tabular}

in any patient with a Glasgow Coma Score (GCS) of less than 12, any patient with a head injury who is agitated or irritable, patients with chest injuries who have inadequate ventilation or patients with multisystem trauma. Twice weekly audit meetings are carried out to discuss missions attended and difficulties encountered.

PROTOCOL

The decision to perform RSI or other advanced airway procedures is made by the HEMS doctor on scene. Etomidate and suxamethonium are used as the standard induction and short acting muscle relaxant agents. Pancuronium is used to maintain muscle paralysis and propofol, morphine and midazolam are carried for continued sedation. Table 1 shows the standard protocol used for RSI. Monitoring of vital signs is carried out using a Propaq (Protocol Systems) monitor. End tidal $\mathrm{CO}_{2}$ verification of tube position is obtained using an in-line infrared spectophotometric method. If intubation is not possible, surgical cricothyriodotomy (or needle cricothyroidotomy in young children) is recommended. Laryngeal mask airways are not routinely carried by HEMS.

DATA COLLECTION

All prehospital "run sheets" of patients treated during the study period were retrospectively reviewed. Age, sex, mechanism of injury, reason for rapid sequence induction and baseline pulse, blood pressure, oxygen saturation, respiratory rate and Glasgow Coma Score before intubation were recorded. The doses of sedative and muscle relaxant drugs used were documented. Visualisation achieved during laryngoscopy was recorded using the Cormack-Lehane grading system ${ }^{14}$ and use of a gum elastic bougie to assist intubation was noted. Pulse, blood pressure, oximetry and end tidal $\mathrm{CO}_{2}$ recordings at 5 minutes and 15 minutes after intubation and on arrival at hospital were identified from the printed recordings of the monitoring system.

The following events were recorded as intubation mishaps: multiple attempts (defined as the need to insert the larnygoscope more than once to achieve intubation) and repeated drug administration (defined as the need to administer a second dose of an induction or muscle relaxant drug to achieve intubation). The number of patients documented to have blood or vomit present in the posterior pharynx at the time of laryngoscopy was recorded. Failed intubation was defined as the inability to achieve intubation after two attempts. The number of surgical airways performed during the study period was also documented.

\section{DATA ANALYSIS}

Data were compared using $\chi^{2}$ analysis for discrete variables and two tailed Student's $t$ test for continuous variables. The Kruskall-Wallis test was used to analyse non-parametric data. A level of $\mathrm{p}<0.05$ was taken to indicate statistical significance.

\section{Results}

RSI was carried out in 359 patients by 19 doctors, of whom 10 were anaesthetists and nine were emergency physicians. All emergency medicine trainees had completed their obligatory three months anaesthetic secondment and all doctors had completed advanced trauma life support training.

Two hundred and two patients were intubated by anaesthetists and 157 by emergency physicians. The mean (SD) age for patients intubated by anaesthetists was 34.5 (30.0) years and for emergency physicians was 35.7 (33.0) years $(p=0.58)$. A total of 147 men and 55 women were intubated by anaesthetists and 120 men and 37 women by emergency physicians $(p=0.43)$. The reason for RSI is shown in table 2 . There was no significant difference between baseline pulse, systolic blood pressure, respiratory rate or oxygen saturation in patients intubated by anaesthetists or emergency physicians. Patients intubated by anaesthetists had a significantly lower $(p=0.009)$ median GCS of 8 than those intubated by emergency physicians with a median GCS of 10. There was a significant difference $(p=0.003)$ between the numbers of patients intubated by emergency physicians who had a GCS of 12 or more (58 of 157) than by anaesthetists (46 of 202).

Doses of induction agents, muscle relaxants and drugs used for maintenance of anaesthesia did not differ significantly between both groups of doctors.

Anaesthetists reported 192 patients (95\%) to have a Cormack-Lehane grade 1 or 2 laryngoscopy compared with 128 patients $(81.5 \%)$ in the emergency physician group. Emergency physicians recorded a significantly larger number of patients $(18 \%)$ in grades 3 and 4 than anaesthetists $(5 \%)(\mathrm{p}<0.0001)$. A gum elastic bougie was used more often by anaesthetists (122 of 202) than by emergency physicians (80 of 157) $(\mathrm{p}=0.024)$. 
Table 3 Failed intubations

\begin{tabular}{|c|c|c|c|c|}
\hline Doctor & Age/sex & Reason for RSI & Difficulties & Outcome \\
\hline Anaesthetist & 65 male & Isolated head injury & Anatomical difficulty & Surgical airway \\
\hline Anaesthetist & 19 male & Multisytem trauma with head injury & Blood ++ in mouth & Surgical airway \\
\hline Emergency physician & 30 male & Burn & Oedematous airway & Surgical airway \\
\hline Emergency physician & 85 male & Burn & Unable to visualise cords due to full thickness neck burn & Surgical airway \\
\hline Emergency physician & 35 male & Isolated head injury & Failed repeat attempt & Self ventilated \\
\hline Emergency physician & 61 male & Isolated head injury & Failed repeat attempt & Self ventilated \\
\hline
\end{tabular}

There was no significant difference in pulse rate, blood pressure, oxygen saturation or end tidal $\mathrm{CO}_{2}$ measurements between the two groups from the time of intubation until arrival at hospital.

The number of intubation mishaps in each group was small. In patients who were successfully intubated anaesthetists commented on an intubation being difficult in eight cases (4\%) and emergency physicians in four $(2.6 \%)$. Repeated attempts at intubation were necessary for one patient $(0.5 \%)$ in the anaesthetic group and three patients $(1.9 \%)$ in the emergency physician group $(p=0.47)$. A second dose of an induction agent or muscle relaxant was given to one patient $(0.5 \%)$ in the anaesthetist group and two patients $(1.3 \%)$ in the emergency physician group $(\mathrm{p}=0.43)$. Two patients treated by anaesthetists developed electromechanical dissociation preceded by a profound bradycardia shortly after intubation. Both occurred in adult men and both patients were successfully resuscitated on scene. One patient in the anaesthetic group was reintubated because of persistent desaturation, thought to be attributable to endobronchial intubation. There were six failed intubations. Details of these patients are given in table 3. A total of eight $(2.2 \%)$ surgical airways were performed during the study period, five $(3.2 \%)$ by emergency physicians and three $(1.5 \%)$ by anaesthetists. This was not statistically significant $(p=0.279)$. Four of the surgical airways were carried out as a result of failure to intubate. Primary surgical airways were performed on the remaining four patients because of anatomical distortion of the face or upper airway, full thickness facial burns or patient entrapment with limited access.

\section{Discussion}

Concern has often been expressed that emergency physicians are not as familiar with sedative and muscle relaxant drugs as anaesthetists with the implication that they may use them in inappropriate doses or circumstances. Our data suggest that similar doses of etomidate and suxamethonium were used by both groups of doctors to allow optimal circumstances for intubation. The use of a variety of induction agents has been studied in emergency departments. ${ }^{1516}$ Etomidate is used by HEMS because it causes less cardiovascular depression than other induction agents. This is particularly important in trauma patients who may be haemodynamically unstable. Patients who were already hypotensive in our series were usually given a reduced dose of etomidate. There were only two recorded cases of significant haemodynamic instability occurring after induction of anaesthesia and both pa- tients had been treated by an anaesthetist. This implies that emergency physicians are able to safely use an anaesthetic induction agent such as etomidate in a potentially unstable group of patients. The only other British study documenting the use of prehospital RSI involved a much smaller number of patients, in whom an array of sedative agents was used. ${ }^{11}$ No details of haemodynamic parameters were given. The use of neuromuscular block in the prehospital setting is well established ${ }^{7810}$ and has been shown to be associated with few complications.

In our series emergency physicians were found to have a significantly larger number of patients with a grade 3 or 4 laryngoscopy and were less likely to use a gum elastic bougie during intubation. The number of failed/repeat intubations was three times higher in the emergency physician group, which although not statistically significant may be clinically significant.These findings suggest that emergency physicians found intubation more difficult than anaesthetists in the uncontrolled prehospital environment. It could be speculated that emergency physicians are less skilled than anaesthetists at the technique of intubation. A group of emergency physicians previously reported difficulty intubating $65 \%$ of trauma patients in the prehopsital setting. ${ }^{11}$ These patients all had a hard cervical collar in situ. Although the emergency physicians in our series seemed to experience more difficulty performing intubation than anaesthetists, the number of repeated attempts at intubation was $<2 \%$, which is much lower than in previously documented reports. $^{917}$ We routinely loosen the collar before intubation, maintaining in line cervical immobilisation from below, which may contribute to our lower complication rate. Performing RSI on patients with potential neck injury makes it difficult to achieve the optimal intubation position. These patients have also been noted to have blood or vomit in the posterior pharynx at the time of laryngoscopy, which Singer et al quotes as a reason for an increased number of intubation mishaps.

Surgical airways were performed on a total of eight patients $(2.2 \%)$, which is much less than the $7.7 \%$ incidence previously reported from the same centre ${ }^{18}$ and other prehospital series. ${ }^{19}{ }^{20}$ Four of these were carried out as a consequence of failure to intubate. The number of failed intubations by emergency physicians was double that of anaesthetists and again may represent clinical if not statistical significance. In two of these cases, both patients with isolated head injuries, the emergency physician elected to allow the patient to self ventilate and was able to complete the transfer to hospital without any subsequent problems. This is clearly not ideal as the main 
reason for attempting to perform RSI on this group of patients is to reduce the risk of secondary brain insults. It could also be argued however that to delay on scene to perform a surgical airway in such patients would also be inappropriate if anaesthetic support is available at a nearby hospital. Our results suggest that emergency physicians only performed surgical airways on patients requiring a definitive airway before transfer to hospital.

No difference between anaesthetists and emergency physicians was identified in haemodynamic parameters, oximetry or end tidal $\mathrm{CO}_{2}$ monitoring at any of the recorded time intervals after intubation. This suggests that emergency physicians are able to use sedative drugs appropriately with no evidence that patients are inadequately sedated in the presence of long acting neuromuscular block. It has been suggested that endobronchial intubation accounts for the majority of cases of arterial desaturation. ${ }^{2122}$ Only one patient in our series was thought to have an endobronchial intubation. Although a small number of other patients desaturated after intubation it was not felt this was attributable to endobronchial intubation but to other underlying disease.

Unlike other reported series, ${ }^{23}$ there were no documented intubation difficulties or mishaps in the group of 80 paediatric patients treated. No reported cases of bradycardia occurred despite the lack of premedication with atropine. ${ }^{1}$ Emergency physicians were able to perform RSI on injured children without experiencing any problems.

It was interesting to note that a significantly larger number of patients with a GCS of 12-15 were intubated by emergency physicians. This may suggest that emergency physicians were able to make an informed decision to anaesthetise a group of patients who were relatively alert, but would benefit from controlled ventilation, implying confidence in their training and ability to perform RSI. ${ }^{24}$ It could also be suggested that anaesthetists were more confident of their ability to intervene in a crisis, should the patient's conscious level suddenly deteriorate. Assuming the degree of difficulty of intubation was distributed randomly, there should be no difference in intubation success or mishap between anaesthetists or emergency physicians. Our results suggest that emergency physicians seemed to have more difficulty performing RSI with a clinical if not statistical significant increase in the number of intubation mishaps. This is hardly surprising given the difference in experience between the two groups. Most of the emergency physicians had completed 6 to 12 months of anaesthetic training compared with the anaesthetists who had completed four to six years training. The aim of our study was to see if emergency physicians were able to safely perform RSI in the prehospital setting. It was therefore reassuring to see that the number of failed intubations and intubation mishaps by emergency physicians was much lower than in previously reported series. We are not suggesting that emergency physicians are as skilled as anaesthetists at performing RSI but suggest that appropriately trained emergency physicians can perform prehospital RSI with very few complications.

\section{STUDY LIMITATIONS}

One potential difficulty with our retrospective study was that recording of intubation difficulties and mishaps varied between run sheets scrutinised. We may therefore be under reporting the number of patients with repeated attempts at intubation. The printed Propaq recordings are also subject to artefact because of patient movement and spurious oximetry readings may be caused by adverse weather conditions. We were unable to obtain follow up data on pulmonary complications after intubation as patients were taken to a large number of hospitals rather than to one centre. The complication rate is so small in both groups of doctors that a much larger series of patients would need to be studied prospectively to show a statistically significant difference between the groups.

\section{Conclusions}

The data suggest that adequately trained emergency physicians are able to safely use sedative and muscle relaxant drugs in prehospital trauma patients. We suggest that emergency physicians can safely perform rapid sequence induction of anaesthesia and intubation in the prehospital setting.

Contributors

Catherine Mackay designed the study, coordinated data collection, completed statistical analysis and was responsible for writing the paper. Jane Terris participated in data collection and analysis and edited the paper. Tim Coats participated in the design of the study protocol, discussed core ideas and interpretation of the findings and edited the paper. $\mathrm{He}$ acts as guarantor for the paper.

Conflicts of interest: none.

Funding: none.

1 Yamamoto LG. Rapid sequence anaesthesia and advanced airway management in pediatric patients. Emerg Med Clin North Am 1991;9:611-38.

2 Talucci RC, Shaikh KA, Schwab CW. Rapid sequence induction with oral endotracheal intubation in the multiply injured patient. Am Surg 1988;54:185-7.

3 Ampel L, Hott KA, Silaff GW, et al. An approach to airway management in the acutely head injured patient. $\mathcal{F}$ Emerg management in the

4 Walls RM. Rapid sequence intubation in head trauma. Ann Emerg Med 1993;22:1008-13.

5 Sakles JC, Laurin EG, Rantapaa AA, et al. Airway management in the emergency department : a one year study of 610 tracheal intubations. Ann Emerg Med 1998;31:325-32.

6 Tayal VS, Riggs RW, Marx JA, et al. Rapid-sequence intubation at an emergency medicine residency : success rate and adverse events during a two year period. Acad Emerg Med 1999;6:31-7.

7 Syverund SA, Borron SW, Stover DL, et al. Prehospital use of neuromuscular blocking agents in a helicopter ambulance program. Ann Emerg Med 1988;17:236-42.

8 Hedges JR, Dronen SC, Feero S, et al. Succinylcholineassisted intubations in prehospital care. Ann Emerg Med 1988;17:469-72.

9 Sing RF, Rotondo MF, Zonies DH, et al. Rapid sequence induction for intubation by an aeromedical transport team a critical analysis. Am f Emerg Med 1998;16:598-602.

10 Murphy-Macabobby M, Marshall WJ, Schneider C, et al. Neuromuscular blockade in areomedical airway management. Ann Emerg Med 1992;21:664-8.

11 Graham CA, Meyer AD. Prehospital rapid sequence induction of anaesthesia. F Accid Emerg Med 1997;14:219-21.

12 Heath KJ, Samra GS, Davies GE, et al. Blood pressure changes in head-injured patients during prehospital anaesthesia with propofol. Injury 1994;25:B7-8.

13 Coats TJ, Newton A. Call selection for the Helicopter Emergency Medical Service: implications for ambulance control. F R Soc Med 1994;87:208-10.

14 Cormack RS, Lehane J. Difficult tracheal intubation in obstetrics. Anaesthesia 1984;39:1105-11.

15 Sivilotti MLA, Ducharme J. Randomised double-blind study on sedatives and haemodynamics during rapid sequence intubation in the emergency department: the SHRED study. Ann Emerg Med 1998;31:313-24.

16 Bergen JM, Smith DC. A review of etomidate for rapid sequence intubation in the emergency department. F Emerg Med 1997;15:221-30. 
17 Adnet F, Jouriles NJ, Le Toumelin P, et al. Survey of out-ofhospital emergency intubations in the French prehospital medical system

18 Xeropotamos NS, Coats TJ, Wilson AW. Prehospital surgical airway management: 1 year's experience from helicopter emergency medical service. Injury 1993;24:22-4

19 Jacobson LE, Gomez GA, Sobieray RJ,et al. Surgical cricothyroidotomy in trauma patients: analysis of its use by paramedics in the field. $\mathcal{F}$ Trauma 1996;41:15-20.

20 Leibovici D, Fredman B, Gofrit AN, et al. Prehospital cricothyroidotomy by physicians. Am 7 Emerg Med 1997;15:91-3.
21 Szekely SM, Webb RK, Williams JA, et al. Problems related to the endotracheal tube: an analysis of 2000 incident reports. Anesth Intens Care 1993;21:611-16.

22 McCoy EP, Russell WJ, Webb RK. Accidental bronchial intubation. Anaesthesia 1997;52:24-31.

23 Sing RF, Reilly PM, Rotondo MF, et al. Out-of-hospital rapid-sequence induction for intubation of the pediatric patient. Acad Emerg Med 1996;3:41-5.

24 Schwartz DE, Matthay MA, Cohen NH. Death and other complications of emergency airway management in critically ill adults. Anesthesiology 1995;82:367-76. 